\title{
Comparison of the predictive utility of Revised Trauma Score, Emergency Trauma Score, and Glasgow Coma Scale-Age-Pressure scores for emergency department mortality in multiple trauma patients
}

\author{
Melis EFEOGLU SACAK ${ }^{1}$ (D), Haldun AKOGLU² (D), Ozge ONUR ${ }^{2}$ (D), Arzu DENIZBASI ${ }^{2}$ \\ ${ }^{1}$ Department of Emergency Medicine, Marmara University, Pendik Training and Research Hospital, Pendik, Istanbul, Turkey. \\ ${ }^{2}$ Department of Emergency Medicine, School of Medicine, Marmara University, Pendik, Istanbul, Turkey.
}

Corresponding Author: Melis EFEOGLU SACAK

E-mail: melisefeoglu@gmail.com

Submitted: 29.04.2020 Accepted: 05.07.2020

\section{ABSTRACT}

Objective: In this study, we aimed to compare the utility of Revised Trauma Score (RTS), Glasgow Coma Scale, Age, and Systolic Blood Pressure (GAP) scores, and Emergency Trauma Score (EMTRAS) in multiple trauma patients for the prediction of mortality in the emergency department (ED).

Materials and Methods: In this observational diagnostic accuracy study, a consecutive convenience sample of all adult patients (older than 16 years) with multiple trauma (injuries confined to at least two body regions) admitted to the trauma bay of the ED during the shifts of the researchers was used. Presence of ED mortality was recorded, and RTS, EMTRAS, and GAP scores were calculated at the analysis stage of this study.

Results: The study sample included 279 multiple trauma patients. Of the 279 patients, 13 (4.7\%) died in the ED. Among the 266 patients who survived to hospital admission, 3 were lost to-follow-up (foreigner patients). In the following 30 days, 28 more patients were lost, 23 in the Intensive Care Unit (ICU) (23/62, 37.1\%), 4 in the wards (4/131, 3.1\%), and 1 after discharge (1/73, 0.1\%). The prognostic accuracies (AUC) of RTS, EMTRAS, and GAP were $0.92,0.94$, and 0.93, respectively, for ED mortality. Conclusion: In this study, all trauma scores performed similar in the ED for the prediction of ED mortality.

Keywords: RTS, EMTRAS, GAP, Trauma, Score, Mortality

\section{INTRODUCTION}

In a pilot study on trauma scoring systems (TSSs) in 1981, five independent predictors of trauma outcome were determined and combined to form the Trauma Score (TS). These five predictors comprise the Glasgow Coma Scale (GCS) score, respiratory rate $(\mathrm{RR})$, respiratory expansion, systolic blood pressure (SBP), and capillary refill [1]. Use of the TS resulted in reliable and accurate prediction of survival after trauma; however, evaluation of capillary refill and respiratory expansion was difficult. Therefore, few years later, the TS was revised by removing these two variables, and the Revised Trauma Score (RTS) was developed, which uses certain coefficients to give higher weight to the GCS score (Table I) [2]. The RTS was accepted by the trauma community worldwide.

Another scoring system is the GCS, Age, and SBP (GAP) scores, which is calculated with the help of each component in its name (Table II) [3]. Compared with RTS, it includes age instead of RR. It is a physiologic TSS that was developed from a multicenter study of 35,732 patients from the Japan Trauma Data Bank (JTDB) who were 16 years or older and had an Injury Severity Score (ISS) of higher than 3. The $c$ statistics of GAP scores in the validation data set $(0.933$ for long-term mortality and 0.965 for short-term mortality) were comparable with those of the RTS (0.919 and 0.966, respectively) [3]. The GAP required fewer parameters and was applicable in the field, and its predictive utility was close to that of the RTS.

Recently, the roles of base deficit (BD), lactate, and traumatic coagulopathy have been better understood in trauma, which led to the idea of using these parameters for the estimation of prognosis. From this idea, the Emergency Trauma Score (EMTRAS) was developed, which is a scoring system that depends on readily available clinical parameters (age, prothrombin time or International Normalized Ratio (INR), and BD) (Table III) [4]. EMTRAS has an accuracy (area under the curve [AUC]) of 0.812 (95\% confidence intervals [CI], 
0.795-0.829) for in-hospital mortality in its derivation and of 0.828 (95\% CIs, 0.792-0.865) in a validation cohort. However, this AUC was considerably lower than that of the other scores, probably because of a missing component, that was, anatomical injury severity [5]. Smaller studies from countries other than Germany, such as Korea (AUC $=0.91 ; 95 \%$ CI, 0.87-0.94) [6], the Netherlands (AUC $=0.92$ and 0.94) [7], and Italy (AUC $=0.80$ ) [8], showed significant variability in the AUCs of EMTRAS.

Trauma scoring systems other than RTS, GAP, and EMTRAS are extremely hard to calculate at the bedside because of the high number of parameters included. Moreover, it is not clear how these scores compare in the emergency department (ED). Although, both EMTRAS and GAP scores may successfully predict mortality, they were not compared to each other in terms of mortality in the ED or survival to hospital admission.

Therefore, in this study, we aimed to compare the utility of RTS, EMTRAS, and GAP scores in multiple trauma patients for the prediction of mortality in the ED.

\section{MATERIALS and METHODS}

This observational diagnostic accuracy study was conducted, in accordance to the statement of Standards for Reporting of Diagnostic Accuracy Studies (STARD) [9], at a Level 1 trauma center with 5000 multiple trauma admissions annually, after the approval by the Institutional Ethics Committee (Approval date and number: 20.09.2013 / 02.2013.0224).

All adult patients (older than 16 years) with multiple trauma (injuries confined to at least two body regions) admitted to the trauma bay of the ED between November 10, 2013, and November 10, 2015, were defined as the source population.

All patients were managed by the attending emergency physician according to the Advanced Trauma Life Support (ATLS) guidelines [10]. During the primary survey, an initial physical examination was performed, vital signs were recorded (temperature, $\mathrm{RR}$, peripheral $\mathrm{O}_{2}$ saturation, $\mathrm{SBP}$, diastolic blood pressure (DBP), and GCS score), and blood was drawn for routine analysis (venous blood gases, hemogram, coagulation panel, blood type and match, electrolytes, blood urea nitrogen, and creatinine). The location(s) (head and neck, trunk, or extremity) and etiology (blunt, penetrating, motor vehicle, fall, etc.) of the trauma were also recorded.

At this point, researchers were alerted, and all data regarding this study were collected at the bedside.

The final study population was a convenience sample consisted of patients admitted during the shifts of the researchers. Patients were excluded from the study if blood for venous gases was not drawn within the first 30 min of the admission.

The primary endpoints for outcome assessment were the mortality rate in the ED. ED mortality was defined as death by any cause while the patient was in the ED (before admitting to a ward, ICU or transfer to another facility).
Revised Trauma Scores, EMTRAS, and GAP scores were calculated according to definitions in previous studies, with the data collected during the ED admission (Tables I, II, III) [2-4].

Table I. Calculation of the RTS score and risk stratification [2]

\begin{tabular}{|c|c|c|c|}
\hline Score & GCS Score & SBP $(\mathbf{m m ~ H g})$ & RR $(/ \mathbf{m i n})$ \\
\hline 4 & $13-15$ & $>89$ & $10-29$ \\
\hline 3 & $9-12$ & $76-89$ & $>29$ \\
\hline 2 & $6-8$ & $50-75$ & $6-9$ \\
\hline 1 & $4-5$ & $1-49$ & $1-5$ \\
\hline 0 & 3 & 0 & 0 \\
\hline
\end{tabular}

RTS: Revised trauma score, GCS: Glasgow coma scale, SBS: Systolic blood pressure, $R R$ : Respiratory rate.

$R T S$ is calculated by adding the weighted sum of the three scores according to the above chart $=([0.9368 \times$ GCS score $]+[0.7326 \times S B P$ score $]+[0.2908 \times R R$ score]).

Table II. Calculation of the GAP score and risk stratification [3]

\begin{tabular}{|l|l|}
\hline Parameter & Score \\
\hline Age $<60$ & 3 \\
\hline SBP $>120 \mathrm{~mm} \mathrm{Hg}$ & 6 \\
\hline SBP $60-120 \mathrm{~mm} \mathrm{Hg}$ & 4 \\
\hline SBP $<60 \mathrm{~mm} \mathrm{Hg}$ & 0 \\
\hline GCS score $(3-15)$ & $3-15$ \\
\hline Total & $3-24$ \\
\hline
\end{tabular}

GAP: Glasgow coma scale (GCS)+Age+Systolic blood pressure (SBP).

Kondo et al. [3] defined the risk categories of the GAP score as follows: Scores from 3 to 10 indicate high ( $>50 \%$ ); 11 to 18 , moderate ( $>5 \%$ and $<50 \%$ ); and 19 to 24 , low risk (<5\%) of death up to 30 days.

Table III. Calculation of the EMTRAS score and risk stratification [4]

\begin{tabular}{|c|c|c|c|c|}
\hline Score & Age & INR & BD & GCS \\
\hline 0 & $<40$ & $<1.25$ & $>-1$ & $13-15$ \\
\hline 1 & $40-60$ & $1.5-2$ & $-1--5$ & $10-12$ \\
\hline 2 & $60-75$ & $2.1-5$ & $-6--10$ & $6-9$ \\
\hline 3 & $>75$ & $>5$ & $<-10$ & -5 \\
\hline
\end{tabular}

INR:International normalised ratio, EMTRAS: Emergency TRAuma Score, BD: Base deficit, GCS: Glasgow coma scale. EMTRAS is calculated by the sum of each parameter, and a total 0-12 is obtained, indicating best to worst prognosis, respectively.

\section{Statistical Analysis}

Continuous variables were summarized as means, standard deviations, and $95 \%$ confidence intervals (CIs) or medians and interquartile ranges according to the distribution of the variable according to normality tests. Categorical variables were summarized as frequencies and percentages. Mean or median values among groups of continuous variables were compared using $t$ test, analysis of variance, and Mann-Whitney $U$ or 
Kruskal-Wallis test. The chi-square test was used to compare categorical variables among groups. Accuracy (AUC), sensitivity, specificity, positive and negative predictive values, and positive $(+\mathrm{LR})$ and negative (-LR) likelihood ratios were calculated from the contingency tables of index tests versus mortality data. The Youden J Index test was used to calculate the optimal threshold value with the highest combined sensitivity and specificity for each index test for each outcome on the Receiver Operating Curves (ROCs). In this study, the accepted Type 1 error was $5 \%$. MedCalc Statistical Software version 18.6 (MedCalc Software bvba, Ostend, Belgium; https://www.medcalc.org; 2016) was used for all analyses.

\section{RESULTS}

A total of 279 multiple trauma patients were enrolled to the convenience sample of this study. The demographics, vital signs, laboratory findings, trauma scores, trauma localizations were summarized and compared according to ED mortality in Table IV. Of the 279 patients, $13(4.7 \%)$ died in the ED. Five of the 13 patients were resuscitated as soon as they arrived in the ED. Among the 266 patients who survived to hospital admission, 3 were lost to-follow-up (foreigner patients). In the following 30 days, 28 more patients were lost, 23 in the ICU $(23 / 62,37.1 \%)$, 4 in the wards $(4 / 131,3.1 \%)$, and 1 after discharge $(1 / 73,0.1 \%)$. Mortality rate after hospital admission was $10.5 \%(n=28 / 263,3$ unknown). Overall 30-day mortality was $14.9 \%(n=41 / 276,3$ unknown). The mortality difference of $5.8 \%$ between before and after hospital admission was statistically not significant $(4.7 \%$ vs $10.5 \%, 95 \%$ CI of the difference $-19.93 \%$ to $22.74 \%, \mathrm{p}=0.5439$ ).

The prognostic utility of the trauma scores is presented in Table V. Screening of other demographic factors, vital signs, and laboratory values revealed that the GCS score was the only prognostic variable, with an AUC higher than 0.90 (Table VI).

Table IV. Demographics of the patients with multiple traumas $(N=279)$

\begin{tabular}{|c|c|c|c|c|}
\hline Variable & $\begin{array}{l}\text { Overall } \\
(\mathrm{n}=279)\end{array}$ & $\begin{array}{l}\text { Survivors } \\
(\mathrm{n}=266)\end{array}$ & $\begin{array}{l}\text { Non-Survivors } \\
(n=13)\end{array}$ & $\mathbf{P}$ \\
\hline Age, median (IQR), years & $37(28-51)$ & $37(28-50)$ & $52(30-71)$ & 0.1616 \\
\hline Male sex, $\mathbf{n}(\%)$ & $239(85.7)$ & $229(86.1)$ & $10(76.9)$ & 0.3580 \\
\hline Occupational accident, $\mathbf{n}(\%)$ & $49(17.6)$ & $47(17.7)$ & $2(15.4)$ & 0.8329 \\
\hline \multicolumn{5}{|c|}{ Vital signs and laboratory findings, median (IQR), $\mathrm{n}=($ survivor/non-survivor) } \\
\hline GCS score & $15(14-15)$ & $15(14-15)$ & $3(3-6)$ & $<0.0001$ \\
\hline SBP, $m m H g(n=264 / 13)$ & $123(110-130)$ & $124(110-130)$ & $65(0-108)$ & $<0.0001$ \\
\hline DBP, $m m H g(n=263 / 13)$ & $78(67-89)$ & $78(68-89)$ & $33(0-58)$ & $<0.0001$ \\
\hline $\mathrm{RR}, / \min (\mathrm{n}=264 / 13)$ & $16(15-20)$ & $16(15-20)$ & $6(0-14)$ & 0.0012 \\
\hline Base excess, $\mathrm{mEq} / \mathrm{L}(\mathrm{n}=192 / 11)$ & $1.2(0.8-4.7)$ & $1.2(-0.9-4.0)$ & $12.9(8.5-19.0)$ & $<0.0001$ \\
\hline $\operatorname{INR}(n=260 / 11)$ & $1.13(1.05-1.22)$ & $1.12(1.05-1.21)$ & $1.46(1.30-1.56)$ & $<0.0001$ \\
\hline Lactate, mg/dL (n=194/13) & $2.6(1.9-3.9)$ & $2.5(1.9-3.6)$ & $10.2(5.2-12.1)$ & $<0.0001$ \\
\hline pCO2, mm Hg (n=193/13) & $\begin{array}{c}43.7 \\
(36.9-49.9) \\
\end{array}$ & $\begin{array}{c}68.3 \\
(44.9-85.7) \\
\end{array}$ & $\begin{array}{c}43.1 \\
(36.9-48.3) \\
\end{array}$ & 0.0034 \\
\hline \multicolumn{5}{|l|}{ Trauma scores, median (IQR) } \\
\hline RTS & $\begin{array}{c}7.841 \\
(7.550-7.841)\end{array}$ & $\begin{array}{c}7.841 \\
(7.841-7.841) \\
\end{array}$ & $\begin{array}{c}3.512 \\
(0-4.624) \\
\end{array}$ & $<0.0001$ \\
\hline EMTRAS & $2(1-3)$ & $1(0-2)$ & $6(5-7.25)$ & $<0.0001$ \\
\hline GAP & $22(21-24)$ & $22(21-24)$ & $9(5.25-14.0)$ & $<0.0001$ \\
\hline \multicolumn{5}{|l|}{ Mortality after ED, n/N (\%) } \\
\hline Mortality in surgery/wards & $4 / 131(3.1)$ & & & \\
\hline Mortality in ICU & $23 / 62(37.1)$ & & & \\
\hline Mortality after discharge & $1 / 73(0.1)$ & & & \\
\hline Mortality total, n/N (\%) & $41 / 276(14.9)$ & & & \\
\hline \multicolumn{5}{|l|}{ Location, $\mathbf{n}(\%)$} \\
\hline Other (soft tissue, peripheral nerves, vessels) & $171(61.3)$ & $161(60.5)$ & $10(76.9)$ & 0.2368 \\
\hline Thorax and mediastinum & $139(49.8)$ & $132(49.6)$ & $7(53.8)$ & 0.7667 \\
\hline Head-neck-face & $117(41.9)$ & $108(40.6)$ & $9(69.2)$ & 0.0415 \\
\hline Extremities & $97(34.8)$ & $89(33.5)$ & $8(61.5)$ & 0.0383 \\
\hline Cranium and central nervous system & $93(33.3)$ & $82(30.8)$ & $11(84.6)$ & 0.0001 \\
\hline Spine & $73(26.2)$ & $71(26.7)$ & $2(15.4)$ & 0.3660 \\
\hline Trunk/abdomen & $62(22.2)$ & $54(20.3)$ & $8(61.5)$ & 0.0005 \\
\hline Pelvis & $49(17.6)$ & $44(16.5)$ & $5(38.5)$ & 0.0429 \\
\hline
\end{tabular}

Three patients of foreign origin were excluded from the study because they were lost to follow-up and their data could not be attained. IQR: Interquartile range, GCS: Glasgow coma sclae, SBP: Systolic blood pressure, DBP: Diastolic blood pressure, RR: Respiratory rate, INR:International normalised ratio, RTS: Revised trauma score, EMTRAS: Emergency Trauma score, GAP: Glasgow coma scale+Age+Blood pressure, ICU:Intensive care unit. 
Table V. Utility of the RTS, EMTRAS, and GAP for ED mortality

\begin{tabular}{|l|c|c|c|} 
& \multicolumn{1}{|c|}{$\begin{array}{c}\text { RTS } \\
(\mathbf{n}=\mathbf{2 7 7})\end{array}$} & $\begin{array}{c}\text { EMTRAS } \\
(\mathbf{n}=\mathbf{1 9 7})\end{array}$ & $\begin{array}{c}\text { GAP } \\
(\mathbf{n}=\mathbf{2 7 7})\end{array}$ \\
\hline AUC $(95 \%$ CI $)$ & $0.92(0.88-0.95)$ & $0.94(0.90-0.97)$ & $0.93(0.90-0.96)$ \\
\hline P & $<\mathbf{0 . 0 0 1}$ & $<\mathbf{0 . 0 0 1}$ & $<\mathbf{0 . 0 0 1}$ \\
\hline $\begin{array}{l}\text { Sensitivity } \\
(95 \% \text { CI })\end{array}$ & $92.3(64.0-99.8)$ & $100.0(69.2-100.0)$ & $92.3(64.0-99.8)$ \\
\hline $\begin{array}{l}\text { Specificity } \\
(95 \% \text { CI })\end{array}$ & $87.5(82.9-91.2)$ & $80.8(74.4-86.1)$ & $86.4(81.6-90.3)$ \\
\hline+ LR (95\% CI) & $7.38(5.2-10.5)$ & $5.19(3.9-7.0)$ & $6.77(4.8-9.5)$ \\
\hline -LR (95\% CI) & $\begin{array}{c}0.088(0.01- \\
0.60)\end{array}$ & 0.0 & $\begin{array}{c}0.089(0.01- \\
0.60)\end{array}$ \\
\hline
\end{tabular}

RTS: Revised Trauma Score, EMTRAS: Emergency Trauma Score, GAP: Glasgow coma scale+Age+Blood pressure, AUC: Area under curve, LR: Likelihood ratios, CI: Confidence interval. (Three patients of foreign origin were excluded from the study because they were lost to follow-up and their data could not be attained.)

Table VI. Prognostic utility of other markers for mortality prediction

\begin{tabular}{|l|c|c|c|}
\hline \multirow{2}{*}{ Variables } & \multicolumn{3}{|c|}{ ED Mortality } \\
\cline { 2 - 4 } & N & AUC (95\% CI) & P \\
\hline Age & 279 & $0.615(0.555-0.672)$ & 0.2221 \\
\hline SBP & 277 & $0.778(0.724-0.826)$ & $\mathbf{0 . 0 0 6 2}$ \\
\hline DBP & 275 & $0.826(0.775-0.869)$ & $\mathbf{0 . 0 0 0 5}$ \\
\hline RR & 277 & $0.764(0.710-0.813)$ & $\mathbf{0 . 0 1 9 7}$ \\
\hline INR & 271 & $0.857(0.810-0.897)$ & $<\mathbf{0 . 0 0 0 1}$ \\
\hline BE & 203 & $0.881(0.829-0.922)$ & $<\mathbf{0 . 0 0 0 1}$ \\
\hline Lactate & 207 & $0.893(0.842-0.931)$ & $<\mathbf{0 . 0 0 0 1}$ \\
\hline pCO2 & 206 & $0.743(0.677-0.801)$ & $\mathbf{0 . 0 2 7 1}$ \\
\hline PTT & 271 & $0.847(0.799-0.888)$ & $\mathbf{0 . 0 0 0 1}$ \\
\hline GCS & 279 & $0.903(0.862-0.935)$ & $<\mathbf{0 . 0 0 0 1}$ \\
\hline
\end{tabular}

The statistically significant $P$ values are denoted in bold. AUC: Area under the curve, SBP: Systolic blood pressure, DBP: Diastolic blood pressure, RR: Respiratory rate, INR:International normalised ratio, BE: Base excess, pCO2: Partial pressure of carbon dioxide, PTT: Partial thromboplastin time, GCS: Glasgow coma scale

The pairwise comparison of the AUCs of the ROCs of scores did not reveal any significant difference (Figure 1).

The ED mortality rates were 9 of 21 (42.9\%), 3 of 28 (10.7\%), and 1 of $228(0.4 \%)$ for high (3-10), moderate (11-18), and low (19-24) GAP risk classes. +LR to rule in ED mortality in the high-risk group was 15.2 (95\% CI, 7.9-29.5), and -LR to rule out ED mortality in the low-risk group was 0.09 (95\% CI, $0.01-0.59)$.

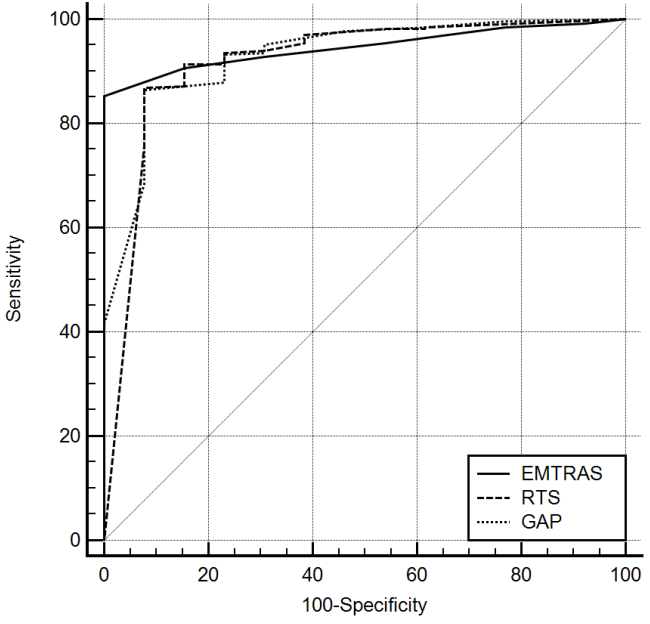

Figure 1. Comparison of the ROC curves of trauma scores regarding ED mortality

\section{DISCUSSION}

In this study, we aimed to compare the utilities of RTS, EMTRAS, and GAP scores in predicting ED mortality in multiple trauma patients, and found that they were highly accurate (AUCs: 0.92, 0.94 , and 0.93 , respectively, Table V), without any significant differences between each other.

The prognostic utility of the RTS was evaluated in countries with lower resources: In 2014, Ahun et al., reported the AUC of RTS for ED mortality as $0.727(\mathrm{P}=0.012)$, in their study in Turkey [11]. In a study from Malaysia, the AUC of RTS for in-hospital mortality was reported to be 0.80 [12]. In another study from Iran, the AUC of RTS for the prediction of in-hospital mortality was reported to be 0.86 (95\% CI, 0.82-0.90) [13]. A recent study from Korea reported the AUC of RTS to be 0.92 (95\% CI, 0.910.93) [14]. In our study, we also found the AUC of RTS for ED mortality as 0.92 . The prognostic utility of RTS seems to be high and similar in middle-income countries, which have a more limited healthcare system with a higher mortality rate in trauma. Research on the utility of the EMTRAS score is insufficient. We were able to locate only four studies that reported on the accuracy of EMTRAS for mortality in the literature. The first study to propose EMTRAS reported the AUCs for in-hospital mortality as 0.81 (95\% CI, 0.80-0.83) and 0.83 (95\% CI, 0.79-0.87) in derivation and validation cohorts, respectively [4]. In a separate validation study, Mangini et al., published the preliminary results of a single-center study of 150 patients and reported an AUC of 0.81 (95\% CI, 0.74-0.87) [15]. In a retrospective, singlecenter study from Korea by Park et al., the predictive values of EMTRAS, RTS, ISS, and Rapid Emergency Medicine Score (REMS) were compared regarding in-hospital mortality in 6905 trauma patients and reported an accuracy of 0.96 for EMTRAS [16]. The last study was a prospective, observational study from Tunisia by Hamed et al. [17]. They evaluated the prognostic performance of trauma scores in terms of mortality at $30^{\text {th }}$ day 
in severe trauma patients and found the AUC of EMTRAS for $30^{\text {th }}$ day mortality as 0.789 , and defined an EMTRAS score of 3 or above as an independent predictor of mortality (adjusted OR 1.80, 95\% CI [1.05-3.08], $\mathrm{p}=0.0033)$. We found the AUC of EMTRAS for ED mortality as 0.94 , and our results are quite similar to research conducted by Park et al. [16].

In 2011, Kondo et al., developed GAP score and calculated the threshold values for low-, moderate - and high-risk categories [3]. They stated that the $c$ statistics for the GAP scores $(0.93$ for long-term mortality and 0.97 for short-term mortality) were better than those for the other trauma scores. Later, Ahun et al. [11], reported that the AUCs of the GAP score for short and long-term mortality as 0.910 and 0.904 , respectively, which were similar to those of the Kondo et al. [3], and Hamed et al. [17], reported an AUC value for GAP as 0.811 for mortality at $30^{\text {th }}$ day, leading score among the compared scores in their study, and defined a GAP score below 20 as an independent predictor of mortality (adjusted OR 1.92, 95\% CI [1.268-2.92], $\mathrm{p}=0.002$ ). In this study, we found the AUC of GAP as 0.93 for ED mortality, and confirmed the previous studies. A pairwise comparison of the ROC values of trauma scores revealed no statistically significant difference in our study.

\section{Limitations}

The limitations of this study should be acknowledged. First, the consecutive sampling of the patient population when the researcher of this study was present in the ED may have created a sampling bias. However, based on the demographics of the study population, it was deemed that this was a good sample of our patient population. Second, patients who died at the scene were eventually excluded, which may have caused, in part, a spectrum bias. However, because this is a study on prognostic scores, the severity of trauma of all patients included in the study is given in detail, which decreases the effect of this bias.

\section{Conclusion}

In this study, all trauma scores performed similar in the ED for the prediction of ED mortality.

\section{Acknowledgment}

We would like to thank the Department of Radiology and Department of Biochemistry for their hard work while preparing the official reports

\section{Compliance with Ethical Standards}

Ethical Approval: The study protocol was approved by the Local Ethics Committee (Approval date and number: 20.09.2013 / 02.2013.0224).

Financial Support: The authors have no relevant financial information to disclose.

Conflict of Interest: The authors have no potential conflicts to disclose.

\section{Authors' contributions}

Initials of the contributing authors were listed in brackets after the relevant parts of the research: Literature search (MES, HA), study design (MES, HA, OO, AD), legislative applications (MES, $\mathrm{HA}$ ), data collection (MES), supervision and quality control (HA, OO, ADA), statistical advice (HA, OO, AD), statistical data analysis (HA), data interpretation (MES, HA), drafting the manuscript (MES, HA). All authors were involved in the writing and critical revision of the manuscript and approved the final version. ME and HA took responsibility for the paper as a whole.

\section{REFERENCES}

[1] Champion HR, Sacco WJ, Carnazzo AJ, Copes W, Fouty WJ. Trauma score. Crit Care Med 1981;9:672-6. doi: 10.1097/00003.246.198109000-00015

[2] Champion HR, Sacco WJ, Copes WS, Gann DS, Gennarelli TA, Flanagan ME. A revision of the Trauma Score. J Trauma [Internet] 1989;29:623-9. doi: 10.1097/00005.373.19890500000017 Accessed on 23.02.2016

[3] Kondo Y, Abe T, Kohshi K, Tokuda Y, Cook EF, Kukita I. Revised trauma scoring system to predict in-hospital mortality in the emergency department: Glasgow Coma Scale, Age, and Systolic Blood Pressure score. Crit Care BioMed Central 2011;15:R191. doi: 10.1186/cc10348

[4] Raum MR, Nijsten MWN, Vogelzang M, et al. Emergency trauma score: an instrument for early estimation of trauma severity. Crit Care Med 2009;37:1972-7. doi: 10.1097/ CCM.0b013e31819fe96a

[5] Wutzler S, Maegele M, Wafaisade A, et al. Risk stratification in trauma and haemorrhagic shock: scoring systems derived from the TraumaRegister DGU $\left(^{\circledR}\right)$. Injury 2014;45 Suppl 3:S29-34. doi: 10.1016/j.injury.2014.08.014

[6] Kim YH, Seo KS, Lee MJ, et al. Application of new trauma scoring systems for mortality prediction in patients with adult major trauma. J Korean Soc Emerg Med 2014;25:447-55.

[7] Joosse P, de Jong W-JJ, Reitsma JB, Wendt KW, Schep NW, Goslings JC. External validation of the emergency trauma score for early prediction of mortality in trauma patients. Crit Care Med 2014;42:83-9. doi: 10.1097/CCM.0b013e31829e53f5

[8] Peris A, Mangini M, Spina R, Franci A, Ognibene A, Zagli G. Application of emergency trauma score in a hub-and-spoke regional system. Br J Anaesth 2013;111:507-8. doi: 10.1093/ bja/aet268

[9] Bossuyt PM, Reitsma JB, Bruns DE, et al. STARD 2015: An updated list of essential items for reporting diagnostic accuracy studies. BMJ 2015;351:h5527 doi: 10.1136/bmj.h5527

[10] American College of Surgeons Committee on Trauma. ATLS Advanced Trauma Life Support. 10 ed. American College of Surgeons; 2018. ISBN 78-0-9968262-3-5. Available at : https:// viaaerearcp.files.wordpress.com/2018/02/atls-2018.pdf Accessed on 11.03.2016

[11] Ahun E, Koksal O, Sığırlı D, Torun G, Donmez SS, Armagan E. Value of the Glasgow coma scale, age, and arterial blood pressure score for predicting the mortality of major trauma 
patients presenting to the emergency department. Ulus Travma Acil Cerrahi Derg 2014;20:241-7. doi: 10.5505/ tjtes.2014.76399

[12] Tan JH, Tan HCL, Noh NAM, Mohamad Y, Alwi RI. Validation of the trauma mortality prediction scores from a Malaysian population. Burns Trauma. BioMed Central 2017;5:37. doi: 10.1186/s41038.017.0102-Z

[13] Nakhjavan-Shahraki B, Yousefifard M, Hajighanbari M, et al. Worthing Physiological Score vs Revised Trauma Score in outcome prediction of trauma patients; a Comparative study. Emerg (Tehran) 2017;5:e31.

[14] Kim SC, Kim DH, Kim TY, et al. The Revised Trauma Score plus serum albumin level improves the prediction of mortality in trauma patients. Am J Emerg Med 2017 ;35:1882-6. doi: 10.1016/j.ajem.2017.06.027

[15] Mangini M, Di Valvasone S, Greco C, et al. Validation of the new proposed Emergency Trauma Score (EMTRAS). Critical Care BioMed Central 2010;14(S1):P252. doi: 10.1186/cc8484

[16] Park HO, Kim JW, Kim SH, et al. Usability verification of the Emergency Trauma Score (EMTRAS) and Rapid Emergency Medicine Score (REMS) in patients with trauma: A retrospective cohort study. Medicine (Baltimore) 2017;96:e8449. doi: 10.1097/MD.000.000.0000008449

[17] Hamed R, Maaref A, Amira F, Aouni H, Mekki I, Jebali A. Prognostic value of scoring tools in severe trauma patients admitted ot the emergency department. Tunis Med 2018 ;96:203-8. 